\title{
A Comparison of the National Institutes of Health Stroke Scale and the Gugging Swallowing Screen in Predicting Stroke-Associated Pneumonia
}

This article was published in the following Dove Press journal:

Therapeutics and Clinical Risk Management

\author{
Phuc Duc Dang $\mathbb{D}^{1,2}$ \\ Minh Hien Nguyen ${ }^{1,2}$ \\ Xuan Khan $\mathrm{Mai}^{3,4}$ \\ Dinh Dai Pham ${ }^{1,2}$ \\ Minh Duc Dang' \\ Dang Hai Nguyen' \\ Van Nam Bui ${ }^{1}$ \\ Duy Ton $\mathrm{Mai}^{5}$ \\ Nhu Binh Do $\mathbb{D}^{6,7}$ \\ Duc Thuan Do (iD ${ }^{1,2}$ \\ 'Department of Stroke, Military Hospital \\ 103, Hanoi, Vietnam; ${ }^{2}$ Department of \\ Neurology, Vietnam Military Medical \\ University, Hanoi, Vietnam; ${ }^{3}$ Respiratory \\ Center, Military Hospital 103, Hanoi, \\ Vietnam; ${ }^{4}$ Department of Tuberculosis \\ and Lung Disease, Vietnam Military \\ Medical University, Hanoi, Vietnam; \\ ${ }^{5}$ Emergency Department, Bach Mai \\ Hospital, Hanoi, Vietnam; ${ }^{6}$ Division of \\ Military Science, Military Hospital 103, \\ Hanoi, Vietnam; ${ }^{7}$ Department of \\ Infectious Disease, Vietnam Military \\ Medical University, Hanoi, Vietnam
}

Correspondence: Duc Thuan Do Tel +84982275220

Email thuandd@vmmu.edu.vn
Background: There have been many scales to predict pneumonia in stroke patients, but they are so complex, making it difficult to apply in practice. Therefore, we conducted this study to assess the role of the National Institutes of Health Stroke Scale (NIHSS) and the Gugging Swallowing Screen (GUSS) in predicting stroke-associated pneumonia (SAP). These scales are routinely used in stroke patients. Therefore, their application in predicting SAP risk will be of high value in clinical practice. There has been no previous study evaluating the effectiveness of SAP risk prediction for each of these scales.

Aim: This study aimed to compare the value of NIHSS and GUSS in SAP prediction and their convenience in clinical practice.

Methods: It was a cohort study. The receiver operating characteristics (ROC) curves were constructed to assess the sensitivity (Se) and specificity $(\mathrm{Sp})$ of the scales. Area under the curves (AUC) were calculated, and we compared them.

Results: NIHSS had a medium value of predictor of SAP with AUC 0.764 (95\% CI $0.735-0.792), 65.4 \%$ Se, $76.5 \%$ Sp. GUSS had good value in predicting SAP with AUC 0.858 (95\% CI $0.833-0.880$ ), $80.5 \%$ Se, $80.1 \%$ Sp. Pairwise comparison of ROCs curves demonstrated that the difference between two AUCs was significant $(\mathrm{p}<0.01)$. Performing GUSS required $24.5 \pm 6.7$ minutes, 2.5 times longer than NIHSS $(9.9 \pm 2.0$ minutes).

Conclusion: GUSS had a better predictive value of SAP than NIHSS. But NIHSS was more convenient in clinical practice because of its simple instrument and quick performance.

Keywords: stroke-related pneumonia, post-stroke pneumonia, pneumonia after stroke, pneumonia prediction

\section{Introduction}

There are many scores for SAP risk prognosis in existence. In 2016 Kishore investigated nine popular scores: ${ }^{1}$ The Pneumonia Score, ${ }^{2}$ Veteran's Health Administration (VHA) cohort score, ${ }^{3}$ A2DS2 (Age, Atrial fibrillation, Dysphagia, Severity of stroke, Sex) score, ${ }^{4}$ PANTHERIS (Preventive Antibacterial Therapy in Acute Ischaemic Stroke) score, ${ }^{5}$ AIS-APS (Acute Ischaemic Stroke-Associated Pneumonia Score) ${ }^{6}$ ICH-APS A (Intracerebral Haemorrhage-Associated Pneumonia Score A), ${ }^{7}$ ICH-APS B, ${ }^{7}$ Pneumonia (PNA) prediction score, ${ }^{8}$ and ISAN (Pre-stroke Independence, Sex, Age, NIHSS) score. ${ }^{9}$ Kishore summed up the components of these scores and found that up to 23 indicators were assessed: age, gender, GCS, NIHSS, dysphagia, dysphasia, increase in systolic blood pressure over $200 \mathrm{mmHg}$, atrial fibrillation, COPD, smoking, diabetes, blood tests, brain 
imaging ... The existence of too many indicators on these scores makes it complicated to apply in practice. In addition, using these scales requires the doctor to spend more time collecting indicators and calculations. Therefore, if we use an available useful tool, it will be convenient for doctors and time-saving. We chose to analyze the two popular scores: NIHSS and GUSS. Our search results on Pubmed, Cochrane, Medline did not show any study on the application of these two scores to predict SAP risk. Hence, we evaluated NIHSS and GUSS as predictors of SAP. Typically, the NIHSS is used to assess the severity of stroke and the GUSS is used to screen for swallowing disorders. In Vietnam, as well as many other countries, these 2 scores are routinely applied to stroke patients. NIHSS and GUSS are available in many languages. We have conducted this study with the aim to compare the value of NIHSS and GUSS in SAP prediction and the convenience of using them in clinical practice.

\section{Materials and Methods}

We enrolled 892 adult patients (aged 18 years and above) admitted to the Department of Stroke, Military Hospital 103 from 2014 to 2017, with acute stroke diagnosis according to WHO criteria. ${ }^{10}$ We diagnosed SAP based on Pneumonia in Stroke Consensus criteria (PISCES): ${ }^{11}$ patients developed pneumonia within 7 days after stroke according to the Centers for Disease Control and Prevention standards. ${ }^{12}$ All patients were excluded from pre-existing pneumonia upon admission by clinical examination and chest X-ray. Patients were continuously monitored, with chest X-ray on the 7th day after onset or immediately whenever symptoms of suspected pneumonia appeared. Patients were divided into 2 groups of SAP and non-SAP.

The severity of stroke was evaluated by NIHSS, a tool used to objectively quantify neurological impairments. It includes the following items: level of consciousness, best gaze, visual fields, facial palsy, arms motor function, legs motor function, limb ataxia, sensory stimulus responses, best language, dysarthria, extinction and inattention. Each item scores a specific ability from 0 to 4 . For each item, a score of 0 indicates a normal function, while a higher score is indicative of more impairment. Total NIHSS score is limited from 0 to 42 (the higher the score, the more severe the stroke). The score is designed to be a simple, valid, and reliable tool that can be administered at the bedside consistently by doctors or nurses. A trainedphysician observed the patient's ability to answer questions and perform activities, then gave ratings for each item. The tool needed to conduct NIHSS is only a sensory needle (Figure 1). NIHSS is available online at http://www.nihstrokescale.org/. The NIHSS scores yielded 4 categories of stroke severity: ${ }^{13}$ very severe (more than 24 points), severe (15 to 24 points), moderate (5-14 points), mild (1-5 points).

We evaluated the swallowing function by using GUSS. ${ }^{14}$ The evaluation process included two stages. In the first stage, we evaluated the indirect swallowing function. In the second stage, we evaluated the direct swallowing function, consisting of 3 segments: Semisolid swallowing trial; liquid
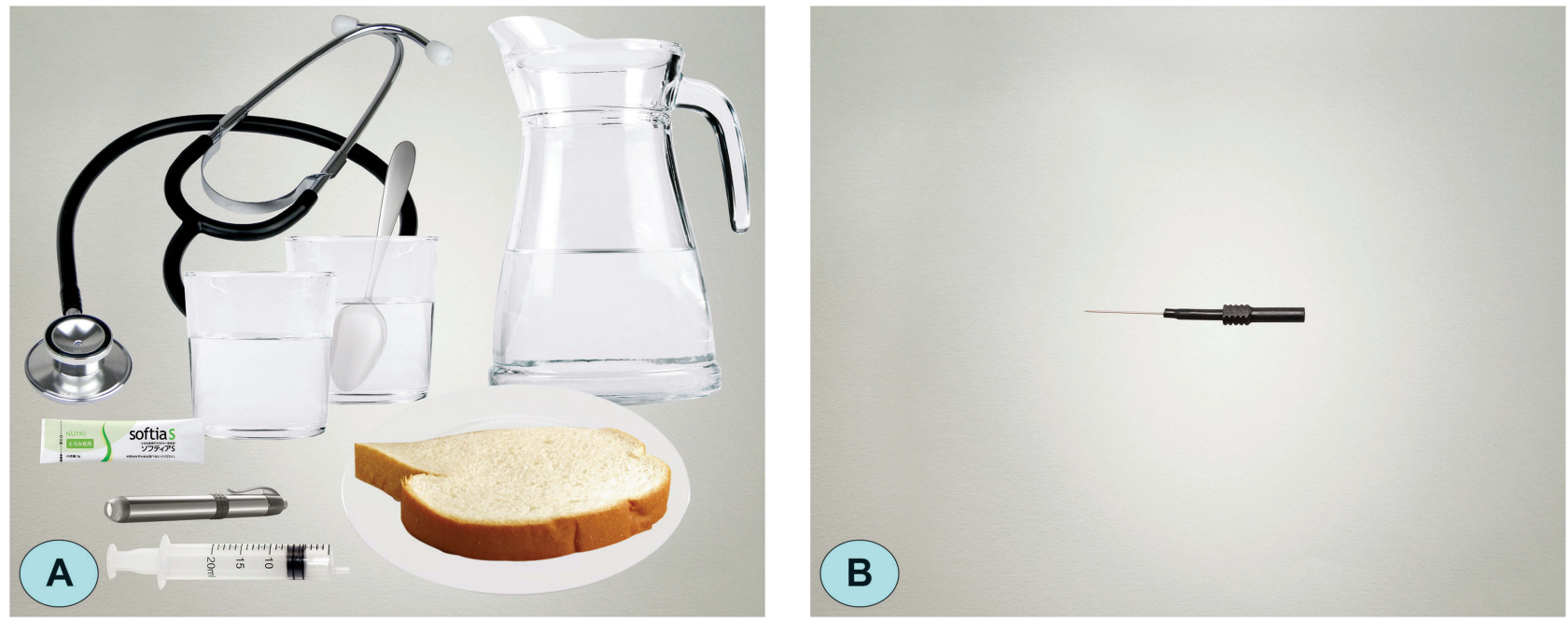

Figure I Materials for GUSS and NIHSS. (A) Materials required for GUSS include 2 cups, a teaspoon, a bottle of drinking water, a stethoscope, a syringe, a penlight, food thickener and a piece of bread. (B) The tool needed to evaluate NIHSS only a sharp needle.

Abbreviations: GUSS, Gugging Swallowing Screen; NIHSS, National Institutes of Health Stroke Scale. 
swallowing trial; solid swallowing trial. Each segment has the highest score of 5. Just only when the maximum score is achieved will the next segment be taken. Total score from 0 (completely incapable of swallowing) to 20 (normal swallowing function). GUSS paper test is available at https:// gussgroupinternational.wordpress.com/guss-sheets/.

The GUSS is divided into 4 categories of severity: ${ }^{14}$ severe ( 0 to 9 points), moderate (10 to 14 points), mild (15 to 19 points), and no dysphagia (20 points). Materials required for GUSS include 2 cups, a teaspoon, a bottle of drinking water, a stethoscope, a $20 \mathrm{~mL}$ syringe, a penlight, food thickener Softia S (Nutri Co., Ltd, Japan) and a piece of bread (Figure 1).

This study was approved by the Ethics Committee of Vietnam Military Medical University (reference No. 06/ 2014/CN-HĐĐĐ). The study was in line with the 1964 Declaration of Helsinki. The informed consent forms were signed by all participants after full explanation.

\section{Statistical Analysis}

We monitored patients until they were discharged and recorded SAP events. In order to determine whether the scores represent the risk for SAP, ROC curves were constructed and compared them by calculating standardized differences between the AUCs (Z-Statistics) using the method presented by DeLong. ${ }^{15}$ In addition, $\mathrm{Sp}$ and $\mathrm{Se}$ of the two scales are also compared at the cutoff decided by DeLong method. The probability of disease was estimated by the odds ratio (OR). All tests were 2-tailed and the statistical significance was determined at $\alpha<0.01$. Statistical analysis was done with Statistical Package for Social Sciences version 15.0.

\section{Results}

From 2014 until 2017, we enrolled 892 stroke patients. Table 1 shows the characteristics of the study population. The mean age of the patients was $66.0 \pm 12.4$ years, of whom 306 patients $(34.4 \%)$ were female, hemorrhagic stroke accounted for $42.4 \%$. The incidence of SAP was $13.8 \%$. Comparing results between SAP and non-SAP groups showed that: SAP group had higher NIHSS $(15.5 \pm 8.7$ and $8.2 \pm 6.0$, respectively, $\mathrm{p}<0.01)$ lower Glasgow Coma Scale (GCS) (12.2 \pm 2.9 and $14.1 \pm 1.8$, respectively, $\mathrm{p}<0.01)$, and lower GUSS $(8.9 \pm 6.6$ and $17.0 \pm 4.9$, respectively, $\mathrm{p}<0.01)$. Among 123 SAP patients, 97 patients $(78.9 \%)$ had new lesions on chest $\mathrm{X}$-rays (New or progressive and persistent infiltrate, consolidation, or cavitation). According to PISCES standard, these patients were diagnosed with "Definite SAP", the remaining patients (accounting for 21.1\%) were diagnosed with "Probable SAP".

NIHSS had medium value in predicting SAP with AUC 0.764 (95\% CI 0.735-0.792), GUSS had good value in predicting SAP: AUC 0.858 (95\% CI 0.833-0.880) (Figure 2). Pairwise comparison of ROC curves using DeLong method

Table I Characteristics of Patients

\begin{tabular}{|c|c|c|c|c|c|}
\hline \multicolumn{2}{|c|}{ Characteristics } & \multirow{2}{*}{$\begin{array}{l}\text { SAP } \\
\text { n (\%) } \\
54(43.9) \\
69(56.1)\end{array}$} & \multirow{2}{*}{$\begin{array}{l}\text { Non-SAP } \\
\text { n (\%) } \\
460(59.8) \\
309(40.2)\end{array}$} & \multirow{2}{*}{$\begin{array}{l}\text { Total } \\
\text { n (\%) }\end{array}$} & \multirow{2}{*}{$\begin{array}{l}\mathbf{p} \\
0.001\end{array}$} \\
\hline Stroke types & $\begin{array}{l}\text { Ischemic } \\
\text { Hemorrhagic }\end{array}$ & & & & \\
\hline Sex & $\begin{array}{l}\text { Female } \\
\text { Male }\end{array}$ & $\begin{array}{l}44(35.8) \\
79(64.2)\end{array}$ & $\begin{array}{l}262(34.1) \\
507(65.9)\end{array}$ & $\begin{array}{l}306(34.3) \\
586(65.7)\end{array}$ & 0.392 \\
\hline Age (years) & $\begin{array}{l}\leq 70 \\
>70 \\
\text { Mean } \pm \text { SD }\end{array}$ & $\begin{array}{l}64(52.0) \\
59(48.0) \\
69.1 \pm 12.1\end{array}$ & $\begin{array}{l}488(63.5) \\
28 I(36.5) \\
65.5 \pm 12.4\end{array}$ & $\begin{array}{l}552(61.9) \\
340(38.1) \\
66.0 \pm 12.4\end{array}$ & 0.017 \\
\hline \multicolumn{2}{|c|}{$\begin{array}{l}\text { GCS (mean } \pm \text { SD) } \\
\text { NIHSS (mean } \pm S D) \\
\text { GUSS (mean } \pm S D)\end{array}$} & $\begin{array}{l}12.2 \pm 2.9 \\
15.5 \pm 8.7 \\
8.9 \pm 6.6\end{array}$ & $\begin{array}{l}14.1 \pm 1.8 \\
8.2 \pm 6.0 \\
17.0 \pm 4.9\end{array}$ & $\begin{array}{l}13.9 \pm 2.1 \\
9.2 \pm 6.9 \\
15.8 \pm 5.8\end{array}$ & $\begin{array}{l}0.001 \\
0.001 \\
0.001\end{array}$ \\
\hline $\begin{array}{l}\text { Diabetes } \\
\text { Smoke } \\
\text { Positive findir }\end{array}$ & rays $^{\mathrm{a}}$ & $\begin{array}{l}34(27.6) \\
10(8.1) \\
97(78,9 \%)\end{array}$ & $\begin{array}{l}71(9.2) \\
60(7.8) \\
0(0.0)\end{array}$ & $\begin{array}{l}105(11.8) \\
70(7.8) \\
97(0.11)\end{array}$ & $\begin{array}{l}0.001 \\
0.845\end{array}$ \\
\hline \multicolumn{2}{|l|}{ Total } & $123(13.8)$ & $769(86.2)$ & $892(100)$ & \\
\hline
\end{tabular}

Note: ${ }^{a} \mathrm{New}$ or progressive and persistent infiltrate, consolidation, or cavitation.

Abbreviations: GCS, Glasgow Coma Scale; NIHSS, National Institutes of Health Stroke Scale; GUSS, Gugging Swallowing Screen; SAP, stroke-associated pneumonia. 


\section{ROC Curve}

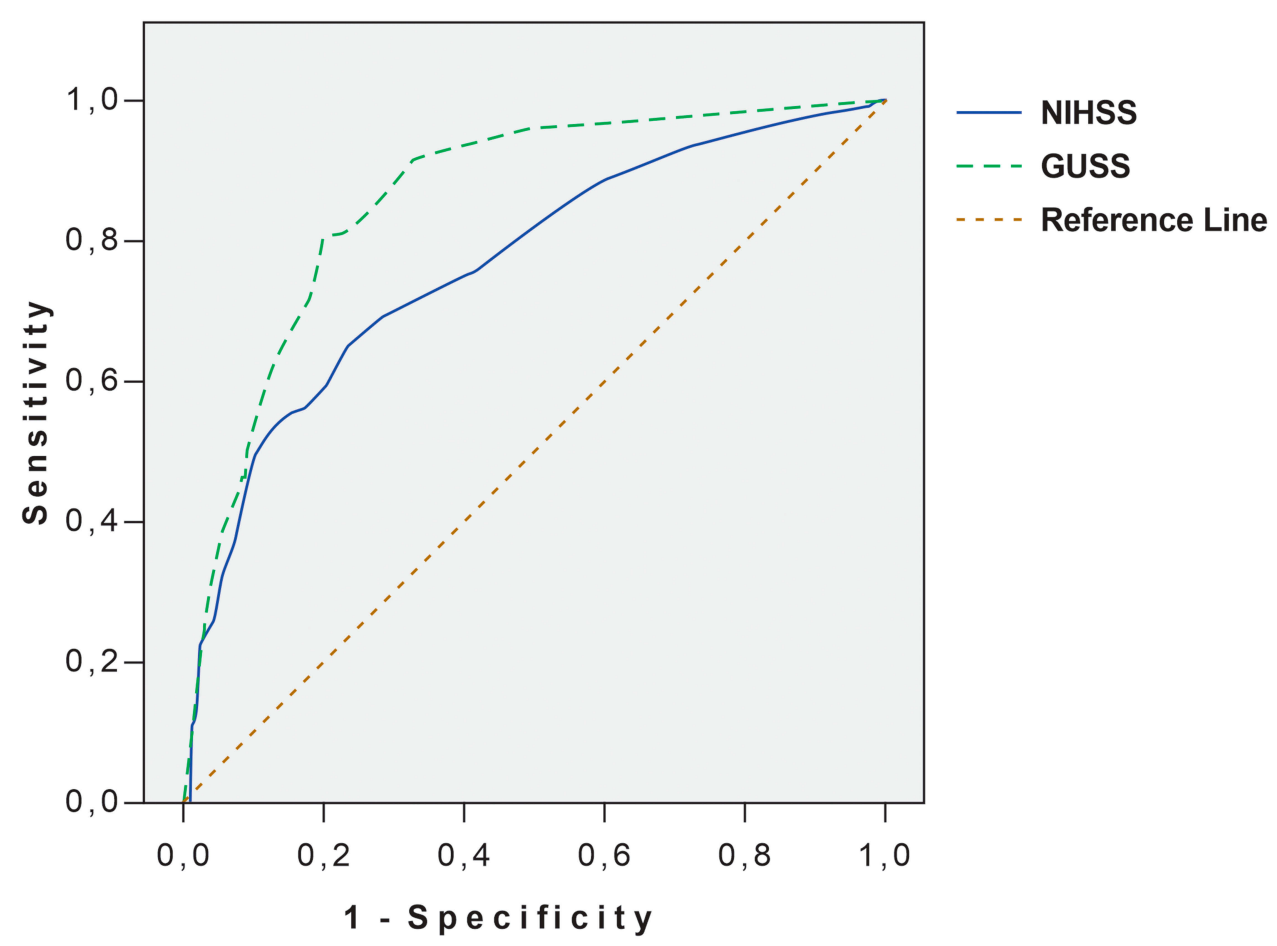

Figure 2 ROC curves for SAP prediction by NIHSS and GUSS.

Abbreviations: ROC, receiver operating characteristics; SAP, stroke-associated pneumonia; NIHSS, National Institutes of Health Stroke Scale; GUSS, Gugging Swallowing Screen.

showed that the difference between AUCs was 0.094 (95\% CI 0.056-0.131), Z-statistics 4.877. The difference was significant $(\mathrm{p}<0.01)$. Two cut-off values of NIHSS and GUSS are 10 and 15 respectively (defined by DeLong method). At the cut-off, NIHSS had $65.4 \% \mathrm{Se}, 76.5 \% \mathrm{Sp}$, and GUSS had $80.5 \% \mathrm{Se}, 80.1 \% \mathrm{Sp}$.

Univariate logistic regression analysis showed that severe and very severe strokes (NIHSS $>15$ ), severe and moderate swallowing disorders (GUSS <15) caused a statistically significant increase in the risk of SAP with OR 8.0 and 11.4, respectively, p $<0.01$ (Table 2).

Table 2 Prediction for the Risk of SAP by Univariate Logistic Regression Analysis

\begin{tabular}{|l|l|l|}
\hline SAP Risk Factors & OR & P \\
\hline Very severe or severe stroke (NIHSS & $8.0(\mathrm{Cl} 95 \%$ & $0.00 \mathrm{I}$ \\
$>$ I5) & $5.3-12.0)$ & \\
Severe or moderate swallowing disorder & $\mathrm{II} .4(7.4-17.5)$ & $0.00 \mathrm{I}$ \\
(GUSS < I5) & & \\
\hline
\end{tabular}

Abbreviations: SAP, stroke-associated pneumonia; NIHSS, National Institutes of Health Stroke Scale; GUSS, Gugging Swallowing Screen; OR, odds ratio; $\mathrm{Cl}$, confidence interval.
Comparing evaluation time for NIHSS and GUSS on each patient showed that performing NIHSS took $9.5 \pm 1.9$ minutes and performing GUSS took $24.5 \pm 6.7$ minutes (Table 3).

\section{Discussions}

Pneumonia is a common subsequent medical complication of stroke. ${ }^{16}$ During the study, 892 patients were enrolled, $123(13.8 \%)$ of them developed SAP.

NIHSS was originally developed in $1989 .{ }^{13}$ In the current American Heart Association/American Stroke Association guidelines, it was recommended as a valid tool to assess stroke severity. ${ }^{17}$ In most stroke units in Vietnam, NIHSS is commonly applied to all patients.

Table 3 Average Time to Evaluate NIHSS and GUSS for Each Patient

\begin{tabular}{|l|l|l|l|}
\hline & Average Scoring Time (Minutes) & Min & Max \\
\hline GUSS & $24.5 \pm 6.7$ & $\mathrm{I}$ & 40 \\
NIHSS & $9.9 \pm 2.0$ & 7 & 15 \\
\hline
\end{tabular}

Abbreviations: NIHSS, National Institutes of Health Stroke Scale; GUSS, Gugging Swallowing Screen. 
Moreover, NIHSS can be conducted quickly (less than 10 minutes) and requires only a sharp object for sensory testing. ${ }^{13}$ In our study, the average time to perform NIHSS was much shorter than that of GUSS $(9.9 \pm 2.0$ minutes, $24.5 \pm 6.7$ minutes, respectively). The problem of saving time on examination and assessment of patients is important, especially in Vietnam and many developing countries, due to limited personnel resources.

GUSS was developed in 2006 at the Landesklinikum Donauregion Gugging in cooperation with the Department for Clinical Neurosciences and Preventive Medicine of the Danube University Krems (Austria). GUSS determines the dysphagia severity and the risk of aspiration in stroke patients. Like NIHSS, GUSS has been translated into many languages, including Vietnamese, which can be easily viewed online at https://gussgroupinternational. wordpress.com/guss-sheets/. Dysphagia occurs in up to two-thirds of stroke patients and can lead to serious complications such as aspiration and pneumonia. ${ }^{18}$ According to Arnold M. et al dysphagic patients suffered more frequently from pneumonia $(23.1 \%$ vs $1.1 \%, \mathrm{p}<0.001) .{ }^{19}$ Screening for dysphagia before the first oral intake of fluid or food after stroke could reduce the risk of aspiration. $^{18,20}$ However, in clinical practice, dysphagia screening is often performed by speech-language therapists, who are not always available in every stroke unit globally. It may lead to delay screening for dysphagia. ${ }^{18}$ In contrast, a trained neurological physician can evaluate NIHSS. Therefore, NIHSS can be scored immediately when patients are admitted to the hospital. Moreover, the materials for assessing GUSS is more complex than the NIHSS (Figure 1). So, perform GUSS requires more preparation time than that of NIHSS.

Our results show that GUSS had AUC of 0.094 higher than that of NIHSS ( 0.858 and 0.764 , respectively), the difference was significant $(\mathrm{p}<0.01)$. Furthermore, GUSS had higher sensitivity in comparison with NIHSS $(80.5 \%$ and $65.4 \%$, respectively). A stroke damages the cerebral hemisphere, cerebellum, or brain stem causing dysphagia. Stroke-caused injuries of the brain may devastate the voluntary function of mastication. Precentral gyrus lesions may cause not only disorder in the lips, tongue, facial motor function but also in peristalsis of the pharynx. Lesions in the brain stem may cause sensation loss of the tongue, mouth and cheek, delay the trigger of the glottis and the pharynx. ${ }^{21}$ Due to dysphagia, foreign objects, food and pathogens easily enter the lower respiratory system. Swallowing disorders is an important mechanism that causes pneumonia in stroke patients. Perhaps that is why the GUSS (direct evaluation of swallowing function) has a higher predictive value of SAP than the NIHSS (a comprehensive evaluation scale of many neurological functions).

\section{Conclusions}

GUSS had a better predictive value of SAP than NIHSS. The AUC of GUSS was 0.094 higher than that of NIHSS, the difference is significant $(p<0.01)$. Moreover, GUSS was more sensitive than NIHSS $(80.5 \% \mathrm{Se}$ and $65.4 \% \mathrm{Se}$, respectively).

However, NIHSS was more convenient in clinical practice because of its quick performance, using just only a common sensory needle. NIHSS could be done 2.5 times faster than GUSS (9.9 minutes versus 24.5 minutes). In addition, NIHSS can be done immediately because sensory needles are always available in every stroke unit. In contrast, using GUSS requires preparation time.

The above results provided more clinical evidence for stroke therapists when considering a suitable scale to predict the SAP risk.

\section{Acknowledgments}

We give many thanks to Nguyen Ngoc Rang for assistance with the data analysis, to Nguyen Minh Man for language correction.

\section{Author Contributions}

All authors made substantial contributions to conception and design, acquisition of data, or analysis and interpretation of data; took part in drafting the article or revising it critically for important intellectual content; gave final approval of the version to be published, and agree to be accountable for all aspects of the work.

\section{Disclosure}

The authors declare that they have no conflicts of interest.

\section{References}

1. Kishore AK, Vail A, Bray BD, et al. Clinical risk scores for predicting stroke-associated pneumonia: a systematic review. Eur Stroke J. 2016;1(2):76-84. doi:10.1177/2396987316651759

2. Kwon H-M, Jeong S-W, Lee S-H, Yoon B-W. The pneumonia score: a simple grading scale for prediction of pneumonia after acute stroke. Am J Infect Control. 2006;34(2):64-68. doi:10.1016/j.ajic.2005.06.011

3. Chumbler NR, Williams LS, Wells CK, et al. Derivation and validation of a clinical system for predicting pneumonia in acute stroke. Neuroepidemiology. 2010;34(4):193-199. doi:10.1159/000289350 
4. Hoffmann S, Malzahn U, Harms H, et al. Development of a clinical score (A2DS2) to predict pneumonia in acute ischemic stroke. Stroke. 2012;43(10):2617-2623. doi:10.1161/STROKEAHA.112.653055

5. Harms H, Grittner U, Dröge H, Meisel A. Predicting post-stroke pneumonia: the PANTHERIS score. Acta Neurol Scand. 2013;128 (3):178-184. doi:10.1111/ane.12095

6. Ji R, Shen H, Pan Y, et al. Novel risk score to predict pneumonia after acute ischemic stroke. Stroke. 2013;44(5):1303-1309. doi:10.1161/ STROKEAHA.111.000598

7. Ji R, Shen H, Pan Y, et al. Risk score to predict hospital-acquired pneumonia after spontaneous intracerebral hemorrhage. Stroke. 2014;45(9):2620-2628. doi:10.1161/STROKEAHA.114.005023

8. Friedant AJ, Gouse BM, Boehme AK, et al. A simple prediction score for developing a hospital-acquired infection after acute ischemic stroke. J Stroke Cerebrovasc Dis. 2015;24(3):680-686. doi:10.1016/j.jstrokecerebrovasdis.2014.11.014

9. Smith CJ, Bray BD, Hoffman A, et al. Can a novel clinical risk score improve pneumonia prediction in acute stroke care? A UK multicenter cohort study. J Am Heart Assoc. 2015;4(1):1-10. doi:10.1161/ JAHA.114.001307

10. World Health Organization. WHO STEPS Stroke Manual: The WHO STEPwise Approach to Stroke Surveillance. 2005.

11. Smith CJ, Kishore AK, Vail A, et al. Diagnosis of stroke-associated pneumonia: recommendations from the pneumonia in stroke consensus group. Stroke. 2015;46(8):2335-2340. doi:10.1161/STROKEAHA.115. 009617

12. Centers for Disease Control and Prevention. CDC/NHSN Surveillance Definition of Healthcare-Associated Infection and Criteria for Specific Types of Infections in the Acute Care Setting. January 1, 2012. Accessed June 25, 2012.

13. Brott T, Adams HP Jr, Olinger CP, et al. Measurements of acute cerebral infarction: a clinical examination scale. Stroke. 1989;20 (7):864-870. doi:10.1161/01.STR.20.7.864
14. Trapl M, Enderle P, Nowotny M, et al. Dysphagia bedside screening for acute-stroke patients: the Gugging Swallowing Screen. Stroke. 2007;38(11):2948-2952. doi:10.1161/STROKEAHA.107.483933

15. DeLong ER, DeLong DM, Clarke-Pearson DL. Comparing the areas under two or more correlated receiver operating characteristic curves: a nonparametric approach. Biometrics. 1988;44(3):837-845. doi:10. 2307/2531595

16. Cugy E, Sibon I. Stroke-associated pneumonia risk score: validity in a French stroke unit. J Stroke Cerebrovasc Dis. 2017;26(1):225-229. doi:10.1016/j.jstrokecerebrovasdis.2016.09.015

17. Powers WJ, Rabinstein AA, Ackerson T, et al. 2018 guidelines for the early management of patients with acute ischemic stroke: a guideline for healthcare professionals from the American Heart Association/American Stroke Association. Stroke. 2018;49(3):e46e99. doi:10.1161/STR.0000000000000158

18. Bray BD, Smith CJ, Cloud GC, et al. The association between delays in screening for and assessing dysphagia after acute stroke, and the risk of stroke-associated pneumonia. J Neurol Neurosurg Psychiatry. 2017;88(1):25-30. doi:10.1136/jnnp-2016-313356

19. Arnold M, Liesirova K, Broeg-Morvay A, et al. Dysphagia in acute stroke: incidence, burden and impact on clinical outcome. PLoS One. 2016;11(2):e0148424. doi:10.1371/journal.pone.0148424

20. Donovan NJ, Daniels SK, Edmiaston J, Weinhardt J, Summers D, Mitchell PH. Dysphagia screening: state of the art. Stroke. 2013;44 (4):e24-e31. doi:10.1161/STR.0b013e3182877f57

21. Martino R, Foley N, Bhogal S, Diamant N, Speechley M, Teasell R. Dysphagia after stroke incidence, diagnosis, and pulmonary complications. stroke. 2005;36(12):2756-2763. doi:10.1161/01. STR.0000190056.76543.eb
Therapeutics and Clinical Risk Management

\section{Publish your work in this journal}

Therapeutics and Clinical Risk Management is an international, peerreviewed journal of clinical therapeutics and risk management, focusing on concise rapid reporting of clinical studies in all therapeutic areas, outcomes, safety, and programs for the effective, safe, and sustained use of medicines. This journal is indexed on PubMed Central, CAS,

\section{Dovepress}

EMBase, Scopus and the Elsevier Bibliographic databases. The manuscript management system is completely online and includes a very quick and fair peer-review system, which is all easy to use. Visit http://www.dovepress.com/testimonials.php to read real quotes from published authors.

Submit your manuscript here: https://www.dovepress.com/therapeutics-and-clinical-risk-management-journal 